\title{
Schipper, Sebastian; Vollmer, Lisa (Hrsg.) (2020): Wohnungsforschung. Ein Reader
}

\author{
Ralf Zimmer-Hegmann
}

Eingegangen: 22. Juli 2021 - Angenommen: 12. August 2021 - Online veröffentlicht: 4. Oktober 2021

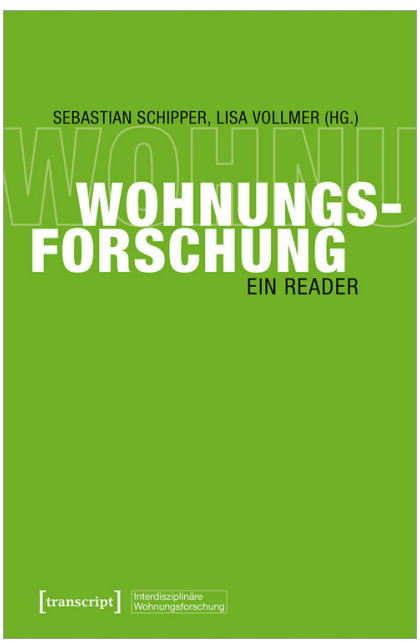

Eines gleich vorweg: Wer sich mit diesem Sammelband (,Reader") zur kritischen Wohnungsforschung neue brandaktuelle Erkenntnisse in der aktuellen wohnungspolitischen Diskussion verspricht, muss sich enttäuscht sehen. Das leistet das Buch nicht, aber das ist auch ausdrücklich nicht der Anspruch. Es geht vielmehr darum, bisherige - zum Teil schon sehr alte - Erkenntnisse in der Wohnungsfrage für die aktuellen wohnungspolitischen Debatten zu erschließen und nutzbar zu machen. Darin liegen der ausgesprochene

Ralf Zimmer-Hegmann, ILS - Institut für Landes- und Stadtentwicklungsforschung, Brüderweg 22-24, 44135 Dortmund, Deutschland

ralf.zimmer-hegmann@ils-forschung.de

(c) (1) (c) 2021 Zimmer-Hegmann; licensee oekom verlag. This Open Access article is published under a Creative Commons Attribution 4.0 International License.
Wert und Nutzen dieses Sammelbandes. Denn grundsätzlich gilt für die sozialwissenschaftliche Forschung - ebenso wie in den Naturwissenschaften -, den stets aktuellsten Forschungsstand auch gemessen an den Publikationsdaten zu berücksichtigen. Dabei unterliegt die sozialwissenschaftliche Forschung durchaus bestimmten zeitlichen Konjunkturen oder gar Zyklen, in denen theoretische und methodische Zugänge und Positionen verschwinden bzw. neue Aufmerksamkeit und Neuformulierung (,Neo-“) erfahren. Insofern kann es in vielen Diskursen hilfreich sein, diesen verschollenen und ,alten ' Erkenntnissen eine höhere Aufmerksamkeit zu widmen. Allerdings erheben Herausgeber und Herausgeberin dieses Sammelbandes nicht nur diesen Anspruch der Erschließung ,alten “ Wissens für einen neuen kritischen Diskurs, sondern erhoffen sich damit auch disziplinäre und häufig fragmentierte Sichtweisen und Diskurse in der deutschen Wohnungsforschung stärker zusammenzuführen und zu vernetzen. Inwieweit dieser Anspruch hinsichtlich Stärkung und Institutionalisierung einer interdisziplinären Wohnungsforschung in Deutschland mit diesem Sammelband eingelöst werden kann, soll am Ende nochmals näher ausgeführt werden.

Der Sammelband fügt 19 bislang schon veröffentlichte „Schlüsselwerke und Überblickstexte“ der kritischen Wohnungsforschung zusammen und gruppiert sie anhand von fünf thematischen Strängen (,Zugriffe“). Sehr hilfreich zum Verständnis der Zielsetzungen und der Struktur des Sammelbandes ist dabei die lesenswerte Einleitung von Sebastian Schipper und Lisa Vollmer. Sie erklärt und unterstreicht den Anspruch nach einer primär kapitalismuskritischen und gleichzeitig auch transformativen Perspektive auf den Gegenstand der Wohnungsforschung, den Wohnraum und das Wohnen. Im Grundverständnis ist der Wohnraum demnach grundsätzlich unter kapitalistischen Verhältnissen als Ware produziert und erfüllt gleichzeitig als Zuhause ein menschliches Grundbedürfnis. In diesem Spannungsverhältnis zwi- 
schen Verwertungsinteressen des Kapitals und den Erfordernissen einer, an den Bedürfnissen der Menschen orientierten, sicheren und bezahlbaren Wohnungsversorgung kommt es zu konflikthaften Auseinandersetzungen und Aushandlungsprozessen. Dabei ist es Aufgabe von kritischer Wissenschaft, progressive wohnungspolitische Alternativen zu analysieren und zu erarbeiten (S. 11-13).

In diesem kritisch-analytischen und transformativ-gestaltenden Grundverständnis werden zunächst sieben Texte unter der Überschrift „Polit-ökonomische Zugriffe“ ausgewählt, die sich vor allem mit der Analyse der kapitalistischen Warenförmigkeit des Wohnens befassen. Sie reichen von älteren Texten (,Klassiker“) aus den 1970er-Jahren über Bodenpreis und Grundrente (Helmut Brede, Barbara Dietrich und Bernhard Kohaupt), die als ,Pionier- und Grundlagenarbeit ' für die kritische Wohnungsforschung gelten, bis zu neueren Texten aus den 2000er-Jahren zur Gentrifizierungsforschung (Inga Jensen und Sebastian Schipper). Die ersten drei Texte in dieser Sektion (Manuel B. Aalbers und Brett Christophers sowie Andrej Holm und Stefan Krät$k e$ ) begründen aus unterschiedlichen Perspektiven die politökonomische und kapitalistische Dimension des Wohnens und damit den Ausgangspunkt und das Grundverständnis für diesen Sammelband. Auch wenn diese drei Texte Redundanzen aufweisen, ergänzen sie sich dennoch gut aufgrund der unterschiedlichen räumlichen und zeitlichen Perspektiven. Abgerundet wird diese Sektion durch zwei englischsprachige Texte (Ulf Torgersen sowie Walter Matznetter), die die international-vergleichenden Debatten und Betrachtungen der Housing Studies auf unterschiedliche Wohlfahrts- und Wohnraumregime ergänzen.

Nach dieser Grundlegung folgen in einer zweiten Kategorie „Historische Zugriffe“ vier Texte, die die Wohnungspolitik in Deutschland in ihrem historisch zeitlichen Verlauf beschreiben und analysieren. Beginnend mit einer breiten historischen Darstellung vom Kaiserreich bis heute (2018) durch Barbara Schönig und Lisa Vollmer über den Klassiker seiner Zeit (1872/1873), Friedrich Engels' „Zur Wohnungsfrage“, bis zu einem stärker spezifischen „Rückblick auf den sozialen Wohnungsbau“ durch Lutz Niethammer (1988). Auch in diesen Beiträgen erfolgt die historische Betrachtung aus einer primär kapitalismuskritischen Perspektive. Sebastian Koch (2016) erklärt in seinem englischsprachigen und international-vergleichenden Beitrag die unterschiedliche Entwicklung und Ausprägung der Wohneigentumsbildung in Deutschland und den USA.

Die dritte Perspektive der "Soziologischen Zugriffe“ knüpft mit dem sehr grundlegenden Text „Soziologie des Wohnens" von Hartmut Häußermann und Walter Siebel (1996) an diese historischen Perspektiven und Darstellungen an, in dem die Entwicklung und Sozialgeschichte des
Wohnens und der Wohnformen in den Zusammenhang mit der Wohnweise von unterschiedlichen Bevölkerungsgruppen und Klassen gebracht wird. In dem zweiten und die aktuelle Debatte um Reurbanisierung aufgreifenden Text konzentriert sich Susanne Frank (2014) auf Abgrenzungsprozesse der „,neuen Mittelschichten“ als soziale Gruppe in - häufig innerstädtischen - räumlichen und kulturellen Enklaven der Stadt.

Nahtlos schließen sich daran die vierten ,(Sozial-)räumlichen Zugriffe“" an, die zunächst in einem breiteren Überblicksbeitrag von Daniela Zupan (2015) die städtebaulichen und stadtstrukturellen Aspekte des Wohnens aus einer primär planungswissenschaftlichen Perspektive beleuchten. Der englischsprachige Beitrag von James DeFilippis und Jim Fraser (2010) knüpft aus einer US-amerikanischen Sicht an den voran gegangenen Mittelschichten-Diskurs an und reflektiert die Debatte um soziale Mischung kritisch. Gerade im Hinblick auf diesen Text hätte man sich auch eine andere ( $\mathrm{Zu}-)$ Ordnung der Beiträge vorstellen können. Gleichzeitig fällt auf, dass in diesem sozialräumlichen $\mathrm{Zu}-$ griff ein dezidierter Beitrag zur residentiellen Segregation fehlt, obwohl das Thema in anderen Beiträgen im Sammelband durchaus angesprochen und ausschnittweise hervorgehoben wird.

Die abschließenden fünften ,Akteurszentrierten Zugriffe“ versammeln im Verständnis einer intervenierenden Wohnungsforschung Beiträge, die die Perspektiven, Positionen und Kämpfe unterschiedlicher Akteurgruppen in der Wohnungsfrage in den Mittelpunkt rücken. Matthias Bernt (2013) beleuchtet zunächst am Beispiel der „Behutsamen Stadterneuerung“ in Berlin die Möglichkeiten, Grenzen und Widersprüche einer kommunalen und staatlich geförderten Städtebau- und Wohnungspolitik. Susanne Heeg (2013) analysiert und kritisiert die Rolle der Immobilienwirtschaft, bei der Wohnungen als Finanzanlage betrachtet werden. Demgegenüber skizziert Klaus Novy (1982) ideengeschichtlich die gegenkulturelle Bewegung der „Wohnungswirtschaftlichen Selbstverwaltung“, also insbesondere gemeinschaftlich-solidarische kommunale und genossenschaftliche Wohnungsversorgungsmodelle. Abschließend wirft Lisa Vollmer (2019) einen recht aktuellen Blick auf die Kämpfe der „Mieter_innenbewegung in Deutschland“.

Insgesamt ist damit eine gute Mischung an Texten der kritischen Wohnungsforschung entstanden, die sowohl interessierten Studierenden eine gute Einführung und Grundlegung in die Themen bietet als auch bezogen auf die Veränderung von wohnungspolitischer Praxis den unterschiedlichen Akteurgruppen der Wohnungspolitik und -wirtschaft einen stärker kritisch reflektierenden Blick auf ihre Praxis vermitteln kann. Insofern kann das Lesen und Aufnehmen 
der Texte durchaus zu einem besseren und stärker analytischen gemeinsamen Verständnis von Wohnungsforschung und Wohnungspolitik über wissenschaftliche Disziplinen und unterschiedliche Akteurgruppen beitragen. Mit einem Preis von 25 Euro ist dieser Sammelband auch verhältnismäßig erschwinglich und damit in der Anschaffung lohnenswert.

\section{Vollständige bibliographische Angaben des} rezensierten Werkes:

Schipper, Sebastian; Vollmer, Lisa (Hrsg.) (2020): Wohnungsforschung. Ein Reader. Bielefeld. = Interdisziplinäre Wohnungsforschung 2. https://doi.org/10.14361/97 83839453513 